\title{
Coordinate regulation of DNA methyltransferase expression during
} oogenesis

\author{
Diana Lucifero ${ }^{1}$, Sophie La Salle ${ }^{1}$, Déborah Bourc'his², Josée Martel ${ }^{1}$, \\ Timothy $\mathrm{H}_{\text {Bestor }}^{2}$ and Jacquetta M Trasler*1
}

Address: ${ }^{1}$ Montreal Children's Hospital Research Institute and Departments of Pediatrics, Human Genetics, and Pharmacology \& Therapeutics, McGill University, Montreal, Quebec, H3H 1P3, Canada and 2Department of Genetics and Development, College of Physicians and Surgeons of Columbia University, New York, New York, 10032, USA

Email: Diana Lucifero - diana.lucifero@bbsrc.ac.uk; Sophie La Salle - sophie.lasalle@mcgill.ca; Déborah Bourc'his - bourchis@ijm.jussieu.fr; Josée Martel - josee.martel@mail.mcgill.ca; Timothy H Bestor - THB12@ columbia.edu; Jacquetta M Trasler* - jacquetta.trasler@mcgill.ca

* Corresponding author

Published: 19 April 2007

BMC Developmental Biology 2007, 7:36 doi:10.1/86/147/-2/3X-7-36

This article is available from: http://www.biomedcentral.com/I47I-2/3X/7/36

(C) 2007 Lucifero et al; licensee BioMed Central Ltd.

This is an Open Access article distributed under the terms of the Creative Commons Attribution License (http://creativecommons.org/licenses/by/2.0), which permits unrestricted use, distribution, and reproduction in any medium, provided the original work is properly cited.
Received: I December 2006

Accepted: 19 April 2007

\begin{abstract}
Background: Normal mammalian development requires the action of DNA methyltransferases (DNMTs) for the establishment and maintenance of DNA methylation within repeat elements and imprinted genes. Here we report the expression dynamics of Dnmt3a and Dnmt3b, as well as a regulator of DNA methylation, Dnmt3L, in isolated female germ cells.

Results: Our results indicate that these enzymes are coordinately regulated and that their expression peaks during the stage of postnatal oocyte development when maternal methylation imprints are established. We find that Dnmt3a, Dnmt3b, Dnmt3L and Dnmtlo transcript accumulation is related to oocyte diameter. Furthermore, DNMT3L deficient I 5 dpp oocytes have aberrantly methylated Snrpn, Peg3 and Igf2r DMRs, but normal IAP and LINE-I methylation levels, thereby highlighting a male germ cell specific role for DNMT3L in the establishment of DNA methylation at repeat elements. Finally, real-time RT-PCR analysis indicates that the depletion of either DNMT3L or DNMT I o in growing oocytes results in the increased expression of the de novo methyltransferase Dnmt3b, suggesting a potential compensation mechanism by this enzyme for the loss of one of the other DNA methyltransferases.

Conclusion: Together these results provide a better understanding of the developmental regulation of Dnmt3a, Dnmt3b and Dnmt3L at the time of de novo methylation during oogenesis and demonstrate that the involvement of DNMT3L in retrotransposon silencing is restricted to the male germ line. This in turn suggests the existence of other factors in the oocyte that direct DNA methylation to transposons.
\end{abstract}

\section{Background}

DNA methyltransferases (DNMTs) catalyze the addition of methyl residues to cytosine bases within CpG dinucleotides to increase the information content of the genome. To date, DNA methylation is the best studied epigenetic modification and is found in the genomes of vertebrates, plants and fungi as well as some species of bacteria and invertebrates [1]. In mammals, the bulk of DNA methylation patterns are established during gametogenesis and early embryogenesis and as such DNA methylation is an 
essential developmental process. DNA methylation is important for the silencing of tandem and interspersed repeat elements and for genomic imprinting and X-chromosome inactivation [2]. Several human disorders are linked to abnormalities in DNA methylation profiles; they include cancer, Rett syndrome, ICF syndrome, as well as genomic imprinting diseases such as Angelman, PraderWilli and Beckwith-Wiedemann syndromes [3].

DNMT enzymes are related by the well-conserved motifs in their catalytic domains and are required for two types of methyltransferase activity, de novo and maintenance methylation. DNMT1 is considered the major maintenance methyltransferase as it is the only DNMT known to exhibit a strong affinity for targeting hemimethylated DNA [4]. Mouse embryos made completely deficient in this enzyme through gene targeting die prior to midgestation and exhibit a 95\% loss of methylation when compared to normal embryos [5]. Homozygous DNMT1deficient embryos have hypomethylated repeat element sequences and biallelic expression of imprinted genes [6]. An oocyte-specific transcript, Dnmt1o, results from the alternative splicing of 5' sex-specific exons within Dnmt1 [7]. Heterozygous embryos derived from Dnmt1o null females show abnormalities in the methylation imprint maintenance and die pre-natally [8]. While DNMT2 is the best conserved of the eukaryotic DNMTs and possesses all the conserved motifs required for DNMT function, DNMT2 has recently been shown to specifically methylate position 38 in tRNA ${ }^{\text {Asp }}[9]$.

In contrast to the predominant maintenance activity of DNMT1 and DNMT1o, studies have revealed that DNMT3a and DNMT3b transfer methyl groups to hemimethylated and unmethylated substrates at roughly equivalent rates [10]. Gene targeting studies have shown that Dnmt3a-/- mice survive a few weeks post-natally, while Dnmt3b-/- embryos die prior to birth [11]. DNMT3L also belongs to the DNMT3 subfamily because of the cysteine rich motif it shares with DNMT3a and DNMT3b. However unlike any of the other DNMTs that have been characterized, DNMT3L lacks the conserved motifs necessary for enzymatic function and therefore lacks DNMT catalytic activity. Instead DNMT3L has been shown to be a potent stimulator of DNMT3a and DNMT3b by changing their conformation and favouring their binding to AdoMet and target sequences [12]. Remarkably, Dnmt3L gene targeting experiments have shown it to be essential for normal spermatogenesis, in particular for meiosis, and required for the methylation of DNA within the differentially methylated regions (DMRs) of imprinted genes in both oocytes and male germ cells [13-16].

Genomic imprinting refers to those genes, numbering roughly 80 to date, which are expressed exclusively from one allele in a parent of origin specific manner. The parental allele specific expression of imprinted genes requires some epigenetic modification to differentially mark the two alleles during germ cell development. Studies have revealed the majority of imprinted genes to be regulated by imprinting control elements (ICEs) which harbour allele specific DNA methylation [17]. In the male germ line, DNA methylation imprint establishment on paternally methylated genes is initiated pre-natally in prospermatogonia [18], while maternally methylated imprinted genes acquire their methylation imprint during the postnatal growth phase of oogenesis [19].

Although DNA methylation is a well studied aspect of imprinted gene regulation, until recently little has been understood about which enzymes are involved in the de novo methylation that marks imprinted genes during gametogenesis. While Dnmt3L gene targeting studies clearly showed this enzyme to be essential for the establishment of methylation imprints in both germ lines [13$16]$, the involvement of DNMT3a and DNMT3b in genomic imprinting remained unclear because of the poor survival and development of DNMT3a and DNMT3b null mice [11]. Conditional germ cell specific knockouts of these enzymes showed that DNMT3a, in a manner similar to DNMT3L, is essential for the de novo methylation of imprinted genes in male and female germ cells [20].

We have previously investigated the temporal dynamics that underlie methylation imprint establishment in oocytes [19]. Here, we examined the expression patterns of the DNMT3 family of enzymes during oocyte development in normal and DNMT-deficient mice in an effort to better understand the role these enzymes play in establishing and/or maintaining DNA methylation patterns during gametogenesis. We used real-time RT-PCR (QRTPCR) to profile the expression of Dnmt3a, Dnmt3b and Dnmt $3 L$ in postnatal oocytes at five different developmental time points and investigated the influence of oocyte diameter on expression patterns. We also determined which DNMT3a isoforms were expressed in growing oocytes. To better understand the role DNMT3L plays in establishing methylation imprints, we studied the methylation profile of multiple imprinted genes in DNMT3L deficient oocytes at $15 \mathrm{dpp}$, the time point when we have previously shown the majority of DNA methylation to be established in oocytes. While DNMT3L clearly has an essential role in ensuring the methylation and silencing of retrotransposons in male germ cells, it is unknown whether it serves the same function in the female germ line. We therefore also investigated the methylation profiles of IAPs and LINE-1 elements in DNMT3L null oocytes. Finally, hypothesizing that these enzymes may be coordinately regulated during development, we moni- 
tored the effect of DNMT3L and DNMT1o depletion on the expression profiles of the other DNMTs.

\section{Results}

Developmental profile of Dnmt3a, Dnmt3b, and Dnmt3L during postnatal oocyte growth

We determined the expression dynamics of DNA methyltransferases in oocytes at five time points spanning the period of postnatal oocyte growth: at $5 \mathrm{dpp}$, oocytes collected were from primordial follicles that had not yet entered the growth phase and measured roughly 10-20 $\mu \mathrm{m}$ in diameter; $10 \mathrm{dpp}$ represented a pool of early-growing oocytes ranging greatly from 20 to $80 \mu \mathrm{m}$ in diameter with roughly half measuring $50 \mu \mathrm{m}$ or less; at $15 \mathrm{dpp}$ our samples consisted of mid-growing oocytes up to $80 \mu \mathrm{m}$ in diameter with only about $5 \%$ of oocytes measuring less than $50 \mu \mathrm{m}$; fully grown GV stage oocytes were collected at $25 \mathrm{dpp}$; and mature ovulated metaphase II (MII) oocytes were isolated from superovulated 8 week old females.

To normalize for variations in RNA input and extraction efficiency, amplification of spiked rabbit $\alpha$-globin was performed for every experiment [21]. The choice of an exogenous normalizer stemmed from the absence of genes known to be expressed at a constant level throughout oogenesis (see Additional file 1). The standard curve method was used to determine the fold changes in expression which were then calibrated to the MII oocyte sample. The QRT-PCR results plotted depict the mean from one representative experiment where each sample was repeated in triplicate.

QRT-PCR results for Dnmt3a, Dnmt3b and Dnmt3L are depicted in Figures 1a, b, and 1c, respectively. Expression of these transcripts increased from 5 dpp oocytes to 25 dpp oocytes, where expression peaked and then decreased slightly in MII oocytes, coinciding with the loss of transcripts which occurs in oocytes after meiotic maturation [22]. In the case of Dnmt3a, the relative expression at 10 dpp was significantly higher than the expression measured at $5 \mathrm{dpp}(\mathrm{p}<0.05)$; while there was no significant difference between the same timepoints for Dnmt3b or Dnmt3L. Dnmt3b expression was significantly upregulated between 5 and 15 dpp ( $<0.05)$, suggesting a more gradual accumulation of transcripts in mid-growing oocytes (Figure 1b). The increase in Dnmt3L expression was highly significant between $10 \mathrm{dpp}$ and $15 \mathrm{dpp}(\mathrm{p}<0.01)$ and showed the greatest fold increase observed for any of the genes or timepoints. With respect to changes occurring during the later stages of oocyte growth, a further significant increase in expression was seen for both Dnmt3a and Dnmt3L between the $15 \mathrm{dpp}$ and full grown GV stage oocytes at $25 \mathrm{dpp}$. This late increase may correlate with previously observed findings that a subset of maternally
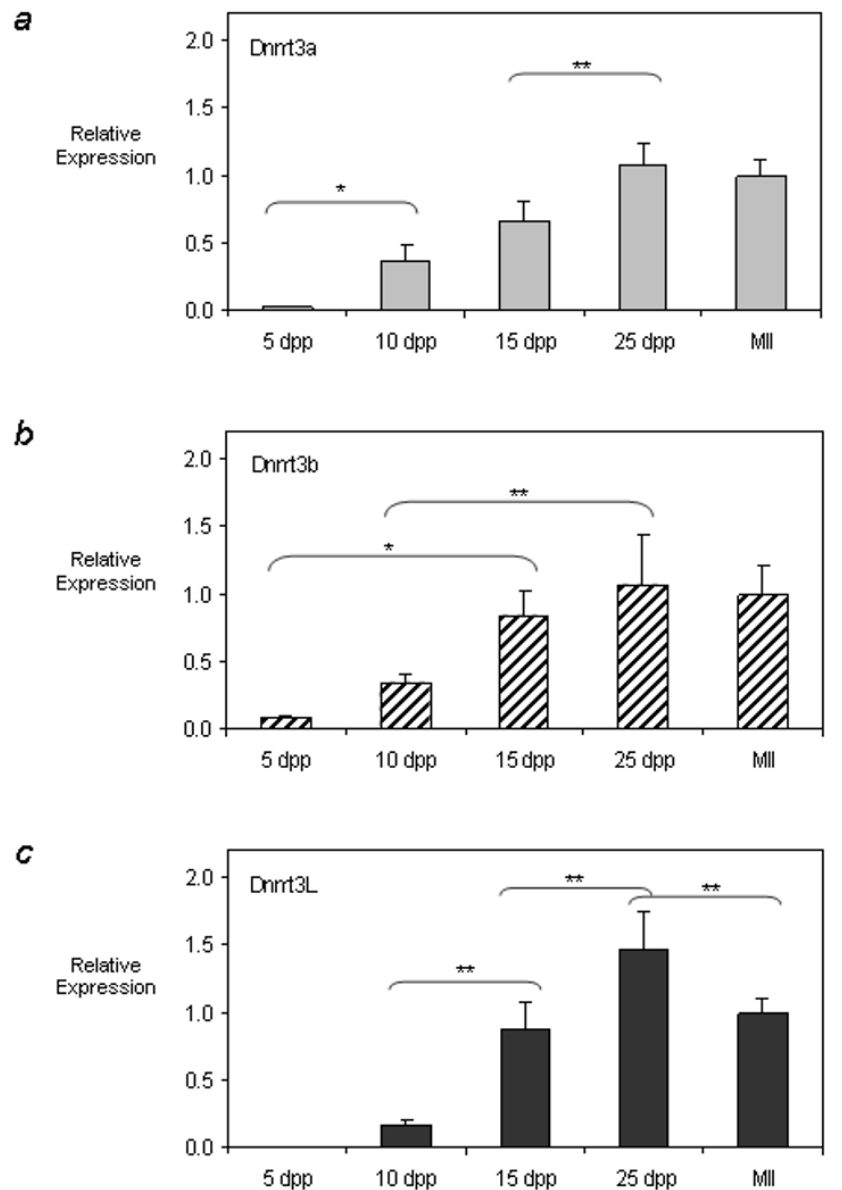

Figure I

Developmental expression profiles of Dnmt3a, Dnmt3b, and Dnmt3L during postnatal oogenesis. QRT-PCR was used to determine the expression profile of a) Dnmt3a (light grey bars) b) Dnmt3b (cross hatch bars) and c) Dnmt3L (dark grey bars) in postnatal oocytes. Relative expression values obtained were normalized to the level of rabbit $\alpha$-globin expression for each sample and were calibrated to the MII oocyte expression value. Representative results from one of the two independent experiments performed are plotted (see Additional file 2 for the second replicate). While the relative abundance of the transcripts varied for each of the timepoints, overall Dnmt3a, Dnmt3b and Dnmt3L expression increased with oocyte growth and peaked in $25 \mathrm{dpp}$ oocytes for each enzyme. Results are presented as mean $\pm S D$. * indicates significance of $p<0.05$; ** indicates significance of $p<0.01$.

methylated imprinted genes acquires methylation late during oogenesis $[19,23,24]$. Another representative replicate showing similar dynamics in expression is included in the additional files (see Additional file 2). 


\section{Relationship of oocyte diameter on Dnmt3a, Dnmt3b, Dnmt3L and Dnmt lo expression}

Having previously determined that oocyte diameter at 15 dpp correlates with DNA methylation levels on the imprinted gene Snrpn [19], we determined whether the expression levels of Dnmt3a, Dnmt3b, Dnmt3L and Dnmt1o also changed with increasing oocyte diameter. Oocytes at $10 \mathrm{dpp}$ were collected and pooled according to their diameter: "small" referring to oocytes 20 to $50 \mu \mathrm{m}$ in diameter and "big" referring to oocytes 60 to $80 \mu \mathrm{m}$ in diameter. For each of the Dnmt genes examined, 60 to 80 $\mu \mathrm{m}$ oocytes showed significantly higher $(\mathrm{p}<0.05)$ levels of transcripts (Figure 2). For both Dnmt3a and Dnmt3b, relative expression levels in "big" oocytes were roughly 4
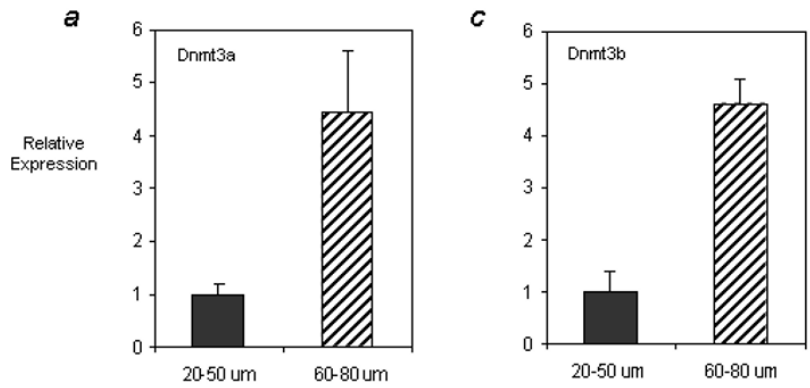

$b$

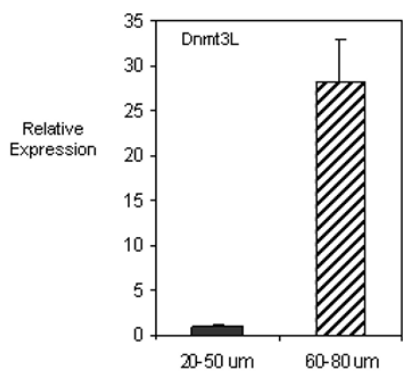

times higher than that in "small" oocytes at $10 \mathrm{dpp}$. Dnmt1o showed a less marked increase with oocyte diameter, with larger oocytes only showing a two-fold increase in expression. The most dramatic increase in transcript level as a result of increased oocyte diameter was seen for Dnmt3L, with the expression in 60 to $80 \mu \mathrm{m}$ oocytes being 28 times that seen in 20 to $50 \mu$ m oocytes.

\section{DNA methylation profile of Snrpn, Peg3, Igf2r, HI9, and retrotransposons IAP and LINE-I in Dnmt3L-/- oocytes}

To better understand the nature of the methylation imprint defect in Dnmt3L-/- oocytes, we investigated the methylation profile of three maternally methylated imprinted genes and one paternally methylated imprinted gene in $15 \mathrm{dpp}$ oocytes (Figure 3a). Using bisulfite sequencing, we analyzed all four genes in one pooled sample of oocytes and found the regions within the Snrpn, Peg3 and Igf2r DMRs sequenced to be unmethylated (analysis of Igf $2 r$ methylation by Fisher's exact analysis showed it to not be significantly different from $0 \%$ ). To control for somatic cell contamination we determined the methylation status of H19, which is methylated in male germ cells but not in oocytes, and found it to be unmethylated in our 15 dpp sample. Surprisingly, although we found the maternally methylated imprinted genes to be unmethylated, CpG sites analyzed within the IAP LTR from the same set of oocytes remained normally methylated (Figure 3b). Fisher's exact analysis using wild-type IAP bisulfite data for this time point [19] showed that the CpG methylation levels obtained between wild type and DNMT3L depleted oocytes were not significantly different $(\mathrm{p}>0.05)$.

Southern analysis with methylation sensitive enzymes using IAP 5' LTR and LINE-1 5' UTR probes also showed these sequences to be normally methylated in mature metaphase II oocytes therefore suggesting that in contrast to male germ cells, Dnmt3L is not required for establishment or maintenance of retrotransposon methylation in the female germ line (Figure $3 \mathrm{c}$ ). While there did appear to be a slight hypomethylation of these sequences in homozygous mutant oocytes, it is important to note that these sequences are not normally fully methylated in wild-type oocytes and when compared to the dramatic differences previously observed in male germ cells [13] the overall pattern of digestion is not significantly different. Somatic cumulus cells also showed no difference in retrotransposon methylation levels (Figure $3 c$ ).

\section{Expression of Dnmt3 transcripts in DNMTIo and DNMT3L depleted oocytes}

In addition to our objective of determining the developmental dynamics of the Dnmt3 genes in postnatal oocytes summarized in Figure 6, we also wished to investigate whether the expression of these transcripts changed in 
a

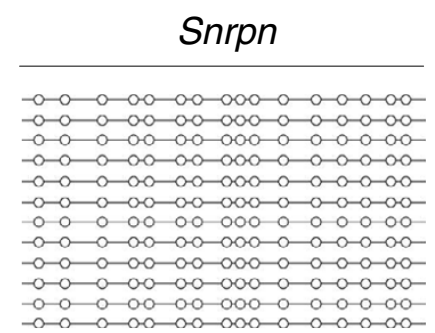

$b$

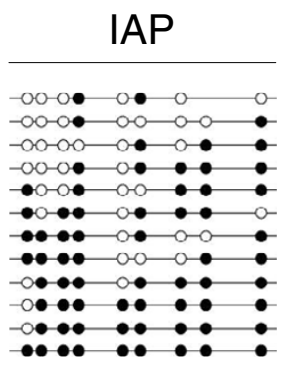

Peg3

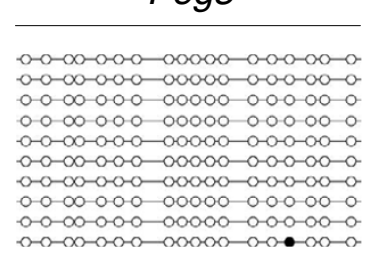

Igf2r

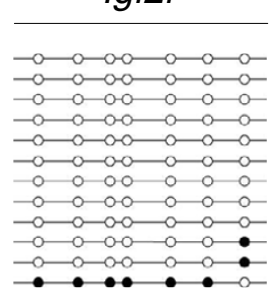

H19

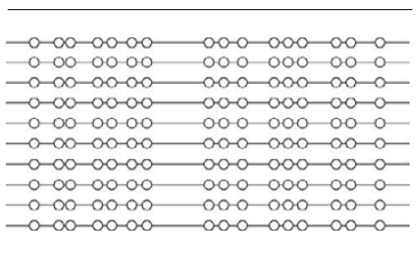

C

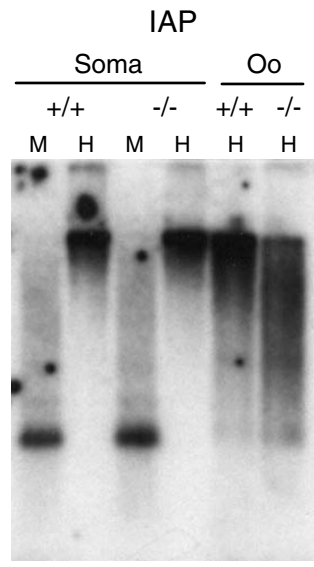

LINE-1

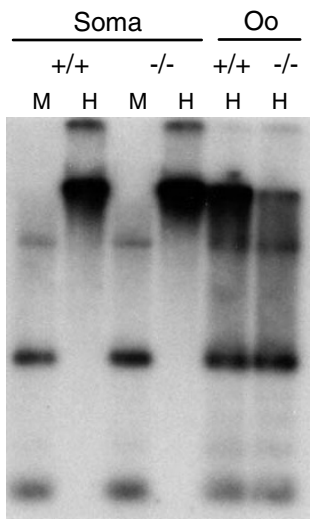

Figure 3

Methylation profile of a) Snrpn, Peg3, Igf2r, HI9 and b,c) IAP and LINE-I repeats in Dnmt3L-/- oocytes. a,b) Bisulfite sequencing results are depicted as follows: each line represents an individual clone; a filled circle indicates a methylated CpG site, an open circle denotes an unmethylated $C_{p G}$ and a missing circle represents a $C_{p G}$ site where the sequencing data were ambiguous. HI 9 methylation served as control for somatic cell contamination. While Snrpn, Peg3 and Igf2r appear to be abnormally unmethylated (a), IAP LTR methylation is unaffected in Dnmt3L homozygous oocytes at I5 dpP (b). c) Methylation sensitive Southern analysis of IAP LTR and LINE-I 5'UTR methylation in Dnmt3L +/+ and -/- cumulus cells (soma) and mature metaphase II oocytes (oo). Lanes headed by $\mathrm{M}$ and $\mathrm{H}$ contain DNA that was cleaved with Mspl and Hpall, respectively. Repeat sequences appear to be normally methylated in DNMT3L depleted oocytes.

response to the depletion of DNMT3L or DNMT1o. We chose to compare expression profiles after depletion of these two DNMTs in particular because the former provides a mouse model where maternal methylation imprints are affected [14], while the latter represents a model where oocyte methylation imprint patterns are unchanged [8]. Again, we used QRT-PCR to look at the expression of Dnmt3a and Dnmt3b in oocytes isolated from heterozygous and homozygous Dnmt $3 \mathrm{~L}$ females at 15 dpp (Figure 4a) and 25 dpp (see Additional file 3). Expression results were calibrated to the value obtained for the heterozygous oocytes and normalized to rabbit $\alpha$ globin transcript levels. When compared to the heterozygous oocytes transcript levels, Dnmt $3 a$ and Dnmt $3 b$ relative expression was up-regulated in response to DNMT3L depletion, increasing roughly 2 - and 3-fold respectively.
The increase in Dnmt3b expression was highly significant ( $\mathrm{p}<0.001)$ while the increase in Dnmt3a expression only approached significance but was reproducible. We also analyzed Dnmt3L expression levels and found heterozygous 15 dpp oocytes to have 360 times the transcript levels as homozygous mutant 15 dpp oocytes ( $p<0.01)$, confirming the purity of our samples (Figure $4 \mathrm{~b}$ ). Analysis of Dnmt3a and Dnmt3b expression changes as a result of DNMT3L depletion in 25 dpp GV oocytes also showed up-regulation of Dnmt3a and Dnmt3b, although not to the same degree as that seen at $15 \mathrm{dpp}$ (see Additional file 3).

These changes at the RNA expression level were also investigated by Western Blot analysis for DNMT3A and DNMT3B in 15 dpp wild-type and homozygous Dnmt3L oocytes (Figure $5 \mathrm{a}, \mathrm{c}$ ). This also allowed us to examine 
a

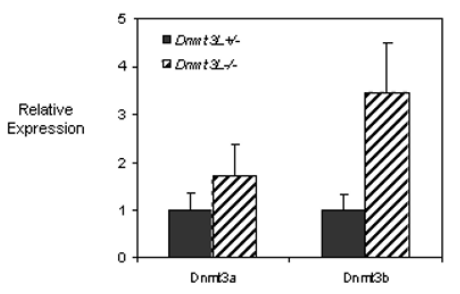

b

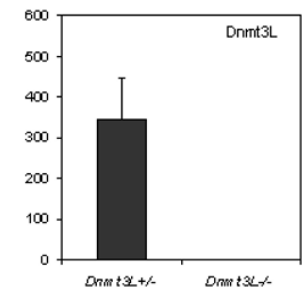

$c$

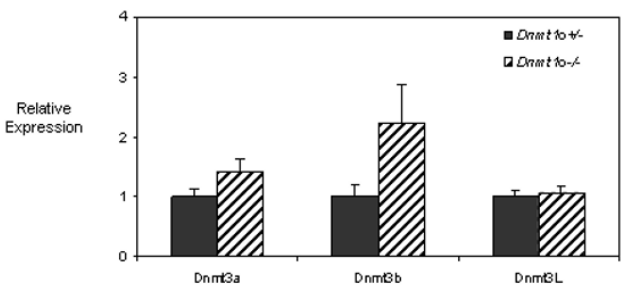

Figure 4

Dnmt3a, Dnmt3b and Dnmt3L expression in DNMT3L deficient 15 dpp oocytes and in DNMT Io deficient 25 dpp GV oocytes. a) QRT-PCR was used to analyze the expression of the de novo DNMT enzymes in Dnmt3L heterozygous (dark grey bars) and homozygous (cross hatch bars) oocytes at I5 dpp. Results for Dnmt3a and Dnmt3b are shown in a) while relative expression of $D n m t 3 L$ is illustrated in b). Dnmt3a and Dnmt3b transcripts are up-regulated in DNMT3L depleted growing oocytes. The differences observed for Dnmt3b and Dnmt3L were statistically significant ( $p<0.0 \mathrm{I})$. c) QRT-PCR was used to analyze the expression of the DNMT enzymes in Dnmt lo heterozygous (dark grey bars) and homozygous (cross hatch bars) $25 \mathrm{dpp}$ GV stage oocytes. While the relative expression of Dnmt3a and Dnmt3b was up-regulated in DNMTIo depleted GV oocytes, Dnmt3L expression remained unchanged. Samples were analyzed in triplicate and relative expression values obtained were normalized to the level of rabbit $\alpha$-globin expression for each sample and were calibrated to the expression in heterozygous oocytes for Dnmt3a, Dnmt3b and Dnmt3L. For Dnmt3L analysis, expression was calibrated to the expression in homozygous oocytes. Results for one experiment are presented as mean \pm SD.

which isoforms of the de novo methyltransferases are present in growing oocytes. As shown in Figure 5a, both DNMT3a isoforms, DNMT3A and DNMT3A2, were detected in 15 dpp growing oocytes. QRT-PCR analysis also showed the transcript variants of Dnmt3a to be of similar abundance in growing oocytes (Figure 5b). Although we saw an increase in Dnmt3a expression in DNMT3L depleted oocytes, a comparable increase in the amount of either DNMT3a isoform in the absence of DNMT3L was not observed when compared to the loading control (see Additional file 4). In contrast, the signifi- cant increase in expression found for Dnmt3b in Dnmt3L /- oocytes was also seen at the protein level (Figure 5c). It appears that the major isoform of DNMT3b in growing oocytes is DNMT3b2, since the band migrates to the same height as the main isoform in type B spermatogonia [25].

We next looked at the changes in expression of Dnmt3a, Dnmt3b, and Dnmt3L in $15 \mathrm{dpp}$ and 25 dpp oocytes isolated from Dnmt1o heterozygous and homozygous mice and found a similar pattern of transcriptional up-regulation seen in the case of DNMT3L depleted oocytes. Two independent experiments each performed in triplicate on two different pools of both heterozygous and homozygous oocytes showed that while Dnmt3L transcript levels were not affected, the de novo DNMTs, Dnmt3a and Dnmt3b, showed an increase in relative expression, with a roughly 1.5 and 2 fold increase respectively in 25 dpp homozygous oocytes (representative results from one experiment are depicted in Figure 4c). Dnmt3b was consistently up-regulated to a greater extent than Dnmt3a at all time points and as a result of either DNMT1o or DNMT3L depletion. At $15 \mathrm{dpp}$, no reproducible change in Dnmt3a, Dnmt3b or Dnmt3L expression levels were observed in DNMT1o null oocytes (see Additional file 5). We confirmed the purity of our germ cell preparations by determining Dnmt1o expression levels in both heterozygote and homozygote DNMT1o deficient oocytes and found expression in the heterozygote oocytes to be roughly 44 times that seen in homozygote oocytes (data not shown).

\section{Discussion \& conclusion}

The establishment and maintenance of DNA methylation patterns on imprinted genes and repeated sequences are essential for normal gametogenesis and embryogenesis in mammals. However, the developmental expression dynamics of the DNMT enzymes responsible for establishing and maintaining these DNA methylation patterns are not well understood, particularly in female germ cells. In the present study, we used QRT-PCR to determine the expression profiles of Dnmt3a, Dnmt3b and Dnmt3L in postnatal oocytes during the period when methylation patterns are being established. We also examined whether expression of these transcripts is related to oocyte diameter, investigated the transcript variants and protein isoforms of Dnmt3a and Dnmt3b found in growing oocytes, and determined the effect of depleting two DNMT enzymes, DNMT1o and DNMT3L, on the relative expression of Dnmt3a, Dnmt3b and Dnmt3L. In addition to exploring the expression dynamics of the de novo enzymes in Dnmt3L homozygous oocytes, we also investigated the methylation defects in these oocytes, with a particular interest in determining the effect of DNMT3L depletion on the methylation status of retrotransposons in female germ cells. 
a

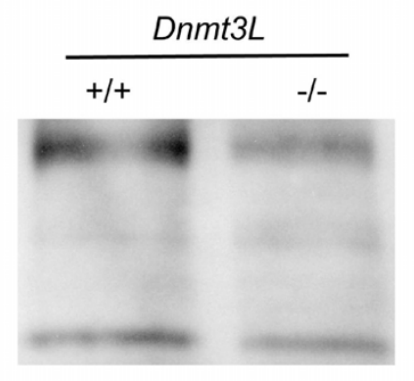

C

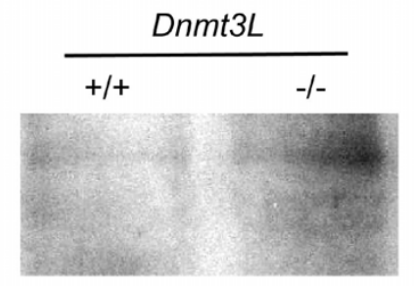

B

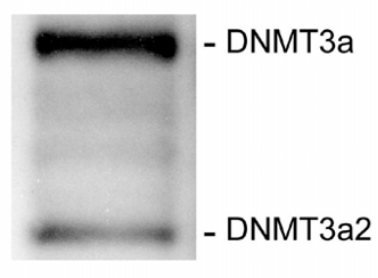

B
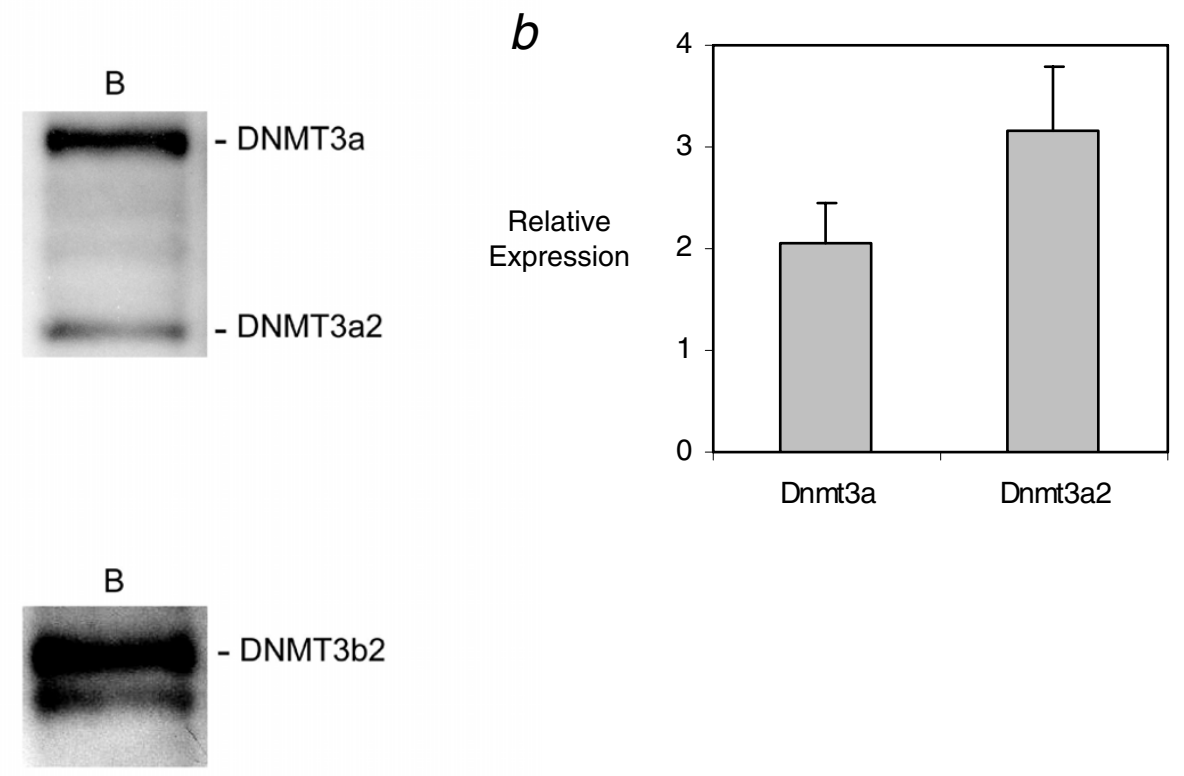

\section{Figure 5}

Protein and transcript variant analysis of Dnmt3a and Dnmt3b in DNMT3L wild type and depleted 15 dpp oocytes. Western Blot analysis of a) DNMT3A and c) DNMT3B was carried out on growing oocytes isolated from Dnmt3L wild-type and homozygous $15 \mathrm{dpp}$ females (280 oocytes/lane). For comparison and isoform identification, the panels on the right labelled 'B' show blotting results for type B spermatogonia (for the loading control see Additional file 4). As suggested by the RNA expression analysis in (b), both DNMT3a and DNMT3a2 are present in growing oocytes and the ratio of these transcripts does not change significantly in Dnmt3L homozygous oocytes. DNMT3b2 is the more abundant isoform in wild-type Dnmt3L oocytes and appears to be upregulated in mutant oocytes as also suggested by the RNA expression data in Figure 4a. b) Relative expression of Dnmt3a and Dnmt3a2 was determined using transcript specific primers. Expression was normalized to I8S. As previously mentioned, both transcripts are expressed in growing oocytes.

Developmental profile of Dnmt3a, Dnmt3b, and Dnmt3L during postnatal oocyte growth and comparison to their expression during spermatogenesis

Several reports have provided some detail on the expression of Dnmt3a, Dnmt3b and Dnmt3L in mouse oocytes. The staining of adult ovaries with X-Gal to reveal the accumulation of a $\beta$-geo marker driven by the endogenous Dnmt3L promoter showed Dnmt3L to be accumulated in growing oocytes $[14,15]$. We have previously used semiquantitative RT-PCR to determine the expression profile of these transcripts during postnatal female germ cell development and showed that they are expressed in growing oocytes [19]. QRT-PCR has been used to determine the expression patterns of these DNMTs in the ovary, and while Dnmt3a and Dnmt3b expression appeared to be stable across the time points examined, Dnmt3L expression clearly peaked post-natally [26]. Still, because of the large population of somatic cells in the ovary, a more thorough assessment of the expression of Dnmt3a, Dnmt3b and Dnmt3L in isolated female germ cells remained necessary to better understand the developmental profile of these DNMTs during oogenesis. Our results suggest that Dnmt3a, Dnmt3b and Dnmt3L have similar expression profiles, with their expression peaking during the crucial period in postnatal oocyte development when maternal methylation imprints are established [19]. Analysis of the differences between the relative expression of the DNMTs suggests that Dnmt3L and Dnmt3a are the most highly upregulated enzymes, consistent with their essential role and cooperation in establishing maternal methylation imprints $[14,15,20,27]$. The increased sensitivity offered by using QRT-PCR has allowed us to detect Dnmt3a, Dnmt3b and Dnmt3L transcripts in MII oocytes which we did not observe in our preliminary analysis [19]. This raises the possibility that these enzymes are stored as maternal transcripts and may play a role in de novo and/or maintenance methylation during preimplantation development.

In addition to studies focusing on female germ cells, the expression of these enzymes in mice has also been investigated during murine spermatogenesis. Depletion of DNMT3a and DNMT3L in male germ cells leads to impaired spermatogenesis [14-16,20]. Expression of $D n m t 3 L$ appears to be confined to a brief perinatal period during which it is highly expressed in non-dividing sper- 


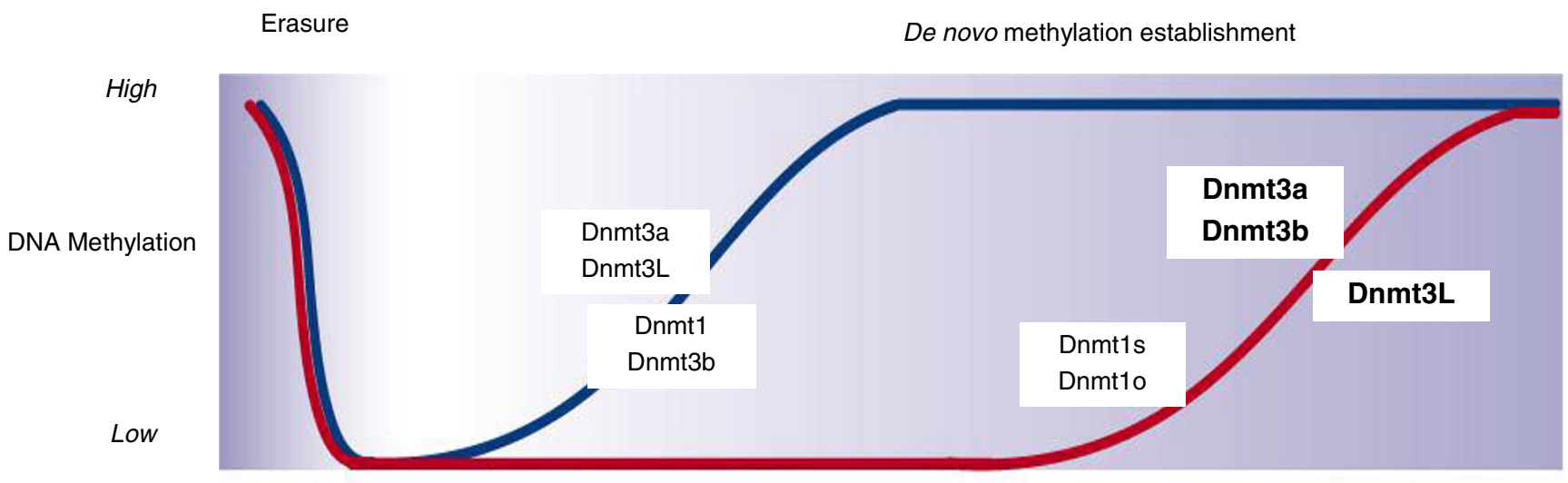

Germ cell development
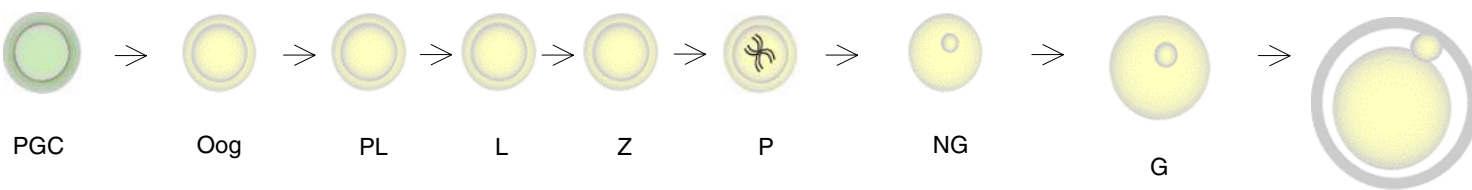

MII

\section{Figure 6}

Onset of DNA methyltransferase expression during mammalian germ cell development. The results presented in this paper as well as observations made for the male germ line $[25,26]$ are depicted here in relationship to the timing of de novo methylation establishment on imprinted genes and repeats during germ cell development. The figure is adapted from [47]. The top panel illustrates the methylation dynamics of maternally and paternally methylated imprinted genes, depicted by the red and blue lines respectively. During gametogenesis the pattern of non-imprinted gene methylation closely resembles that of imprinted genes. The time at which DNA methyltransferases are expressed during germ cell development are indicated with the transcripts examined in this study highlighted in bold. Although expressed at similar stages of oogenesis, the de novo DNA methyltransferases, Dnmt3a and Dnmt3b, are grouped separately from Dnmt3L which does not have DNA methyltransferase activity. The onset of Dnmt lo expression has been previously shown in early growing oocytes [43]. Our findings show that the expression of Dnmt3a, Dnmt3b and Dnmt3L coincide with the establishment of DNA methylation on imprinted genes and repeat sequences in the female germ line. The progression of methylation imprint acquisition during oogenesis is illustrated in the bottom panel and depicted by the red shading above the female germ cells. PGC = primordial germ cell, Oog = oogonia, PL = preleptotene, $\mathrm{L}=$ leptotene, $\mathrm{Z}=$ zygotene, $\mathrm{P}=$ pachytene, $\mathrm{NG}=$ non-growing oocyte $\mathrm{G}=$ growing oocyte, $\mathrm{MII}=$ metaphase II oocyte.

matogonial stem cell precursors [13]. The testis-wide and germ cell specific expression of the DNMT3 transcripts has been examined using a QRT-PCR approach $[25,26]$. In the developing testis, Dnmt3a and Dnmt3L have similar expression profiles with expression peaking pre-natally at $15.5 \mathrm{dpc}$, while Dnmt3b expression peaks post-natally at 6 dpp [26].

Comparing the developmental expression profile of Dnmt3a, Dnmt3b and Dnmt3L in oocytes and the expression patterns previously described in the male germ line, sex-specific differences in the expression of this family of enzymes are apparent (Figure 6). The perinatal expression of both Dnmt3a and Dnmt3L in male germ cells fits well with the timing of methylation pattern establishment during spermatogenesis. Both paternally methylated imprinted genes and repeat sequences begin to acquire methylation pre-natally in prospermatogonia and DNA methylation patterns continue to be established and maintained peri-natally $[18,28-30]$. In contrast, Dnmt3a, $D n m t 3 b$ and Dnmt3L are expressed in postnatal growing oocytes during the period when maternally methylated imprinted genes and repeat elements acquire methylation $[19,23,24,30,31]$. 


\section{Increased Dnmt3a, Dnmt3b, Dnmt3L and Dnmt lo} expression correlated with an increase in oocyte diameter We have previously shown that methylation acquisition within the DMR of at least one imprinted gene is related to oocyte diameter [19]. Hiura et al. (2006) [24] and Bao et al. (2000) [23] also found that a certain threshold in oocyte diameter is necessary for epigenetic modifications to have been thoroughly established in oocytes. Preliminary semi-quantitative RT-PCR analysis suggested to us that the expression of Dnmt3L may be influenced by oocyte diameter [19]. Here, using a QRT-PCR approach we confirmed Dnmt3L expression to be correlated to oocyte diameter and also found Dnmt3a, Dnmt $3 b$ as well as Dnmt1o expression to significantly increase with increasing oocyte diameter. Postnatal oogenesis is a period during which the oocyte grows greatly in volume and accumulates a variety of factors necessary for meiotic maturation and early preimplantation development [32]. The increase in transcription that accompanies this growth period also leads to elevated expression levels of Dnmt3a, Dnmt3b, Dnmt3L and Dnmt1o in fully grown oocytes.

\section{Aberrant methylation of Snrpn, Peg3, and Igf2r but normal methylation of IAP and LINE-I retrotransposons in growing Dnmt3L-/- oocytes}

It has been previously reported that depletion of DNMT3L in the oocyte results in impaired establishment of DNA methylation patterns on imprinted genes inheriting a maternal methylation mark [14,15]. Although abnormal methylation of the Snrpn and Peg1 DMRs was described in mature MII oocytes [14], it remained unknown if different DMRs showed the same methylation defect and if this defect occurred early or late during female germ cell development. Here, we analyzed the methylation profile of Snrpn, Igf2r, and Peg3 in DNMT3L deficient mid-growing oocytes. We have previously observed methylation levels on these 3 genes at $15 \mathrm{dpp}$ to be between 60 and $80 \%$ [19]. In keeping with the previously reported Snrpn and Peg1 results [14], we found Snrpn, Peg3 and Igf2r to be unmethylated in Dnmt3L-/- 15 dpp oocytes, suggesting that DNMT3L is involved in the earliest stages of maternal methylation imprint acquisition.

In addition to analyzing imprinted gene methylation, we also examined the methylation status of the IAP 5' LTR and the LINE-1 5' UTR in both growing and mature oocyte samples and found these retrotransposable elements to be normally methylated when compared to prior analysis of these same regions in oocytes $[19,33]$. While the methylation status of retrotransposons in Dnmt3L homozygous oocytes was unknown, previous experiments suggested these sequences to be unaffected in post-implantation Dnmt3L+/- embryos derived from the fertilization of DNMT3L deficient oocytes [14]. Detailed studies have clearly shown that DNMT3L is required for establishing methylation on LTR and non-LTR retrotransposable elements in the male germ line and that this role for DNMT3L is important for ensuring normal meiosis $[13,16]$. In contrast, our analysis indicates that DNMT3L is not required for the establishment of methylation patterns on IAPs or LINE-1s in oocytes. In contrast to sperm, IAP and LINE-1 elements are not fully methylated in mature wild-type oocytes. This may be additional evidence of some different requirement of the two parental germ lines on methylation-dependent retrotransposon silencing. Together, these observations suggest germ line specific functions for DNMT3L and demonstrate that its involvement in retrotransposon silencing is restricted to male germ cells. Analysis of IAP LTR methylation in DNMT3a depleted oocytes showed these repeats to be hypomethylated [20]. It remains possible that other factors together with DNMT3a are involved in retrotransposable elements methylation during oocyte development. Interestingly, a recent report suggests the involvement of LSH, a member of the SNF2-helicase family of chromatin remodellers, in retrotransposon silencing in female germ cells [34].

\section{Up-regulation of the de novo DNMTs in DNMTIo and DNMT3L depleted oocytes}

In contrast to the Dnmt3L knockout, where genomic imprints are not established [14], depletion of DNMT1o does not affect the acquisition of genomic imprints [8]. To determine whether Dnmt3a, Dnmt3b and Dnmt3L are coordinately regulated or if they can compensate for each other, the expression profiles of these enzymes were compared between heterozygous and homozygous oocytes for both models. QRT-PCR results indicated that in response to DNMT1o depletion, the expression of the de novo DNMTs, Dnmt3a and Dnmt3b, was up-regulated in $25 \mathrm{dpp}$ GV oocytes, while Dnmt $3 L$, which lacks DNA methyltransferase activity, showed no significant change. Dnmt $3 a$ and Dnmt3b were also up-regulated in the 15 dpp Dnmt3L-/oocytes analyzed. While the increase in Dnmt3a expression was modest, Dnmt3b expression was significantly upregulated in both DNMT1o and DNMT3L depleted oocytes with a parallel increase in protein expression observed in DNMT3L depleted 15 dpp oocytes. Similarly, Gius et al. (2004) [35] also observed a three-fold increase in Dnmt3b transcripts in a Dnmt1 knockout cell line. These findings suggest that in the absence of one DNMT, a compensatory feedback mechanism may exist which leads to the up-regulation of $D n m t 3 b$ in an attempt to rescue the defect in de novo and/or maintenance methylation.

It is possible that while such an upregulation may play a role in maintaining DNA methylation on retrotransposons and other repeat sequences in the genome of Dnmt3L-/- mice, it is unable to maintain methylation on 
imprinted sequences due to the absence of DNMT3L. The activation of retrotransposons in the male germ line has been shown to have deleterious consequences on meiosis [13]. IAP transposons also appear to be resistant to active demethylation 'reprogramming' in primordial germ cells and zygotes [36]. In this report the authors speculate that maintaining these elements methylated may prevent their transposition and consequently avert the introduction of deleterious mutations in the genomes of gametes and preimplantation embryos. Therefore, the upregulation of DNMTs may be an additional mechanism which has evolved to ensure the silencing of these elements in the female germline. However, upregulation of a DNMT not normally expressed at high levels at a certain stage of oocyte development could potentially also have negative repercussions and contribute to aberrant methylation patterns in Dnmt knock-out oocytes.

Recent analysis of stochastic imprinting events in the progeny of Dnmt3L-/- females showed that sporadically, in some embryos derived from DNMT3L null oocytes, maternal DMRs were methylated and imprinted expression was normal [37]. The authors suggest that in the absence of DNMT3L, the other DNMTs or additional factors may act to rescue imprint establishment in the germline [37]. It is possible that the up-regulation of Dnmt3b that we observed in Dnmt3L-/- growing oocytes may contribute to this rescue. Interestingly, for Igf2r, one of the sequenced molecules was methylated at 6 of the $7 \mathrm{CpG}$ sites analyzed (Figure 3a). This observation may be reflective of the stochastic imprinting event also observed by Arnaud et al. and may be a result of the rare instance where the upregulation of the other DNMTs was able to rescue the methylation imprint. The overall failure of the DMRs to be methylated during oogenesis in Dnmt3L-/oocytes which are 'over-expressing' Dnmt $3 b$ may be due to the inefficient targeting of the enzymes in the absence of DNMT3L. Previous data have shown maternally imprinted genes in Dnmt3L -/- mature MII oocytes to be unmethylated $[14,15]$. Therefore an alternative possibility to reconcile the results presented here with those described by Arnaud et al. is that in the absence of DNMT3L the DMRs acquire methylation during preimplantation development perhaps as a result of increased expression of Dnmt3a and Dnmt3b which are carried over as maternal transcripts.

Prior studies have pointed to the interaction and interdependence of DNMT enzymes. In vitro studies have suggested that DNMT3L can stimulate de novo methylation catalyzed by DNMT3a at several ICEs [27]. Physical interactions between DNMT3L and the C-terminal domain of both DNMT3a and DNMT3b, as well as interactions between DNMT1 and DNMT3a or DNMT3b have been described [38-40]. Together with these studies, the QRT-
PCR results described here suggest that Dnmt3a, Dnmt3b and Dnmt3L are developmentally and may be coordinately regulated to ensure the proper methylation of both imprinted and non-imprinted sequences in the female germ line.

\section{Methods \\ Oocyte collections and mice}

MII oocytes as well as growing oocytes at 5, 10, 15, $25 \mathrm{dpp}$ were isolated as previously described $[19,41]$. Briefly, oocytes at 5, 10, 15, and 25 dpp were isolated from dissociated ovaries obtained from CD-1 mice (Charles River Canada, St. Constant, QC). The dissection of 5, 10, and 15 dpp ovaries was carried out in PBS, pH 7.2. After transfer to conical tubes containing $2 \mathrm{ml}$ of $3 \mathrm{mg} / \mathrm{ml}$ polyvinylpyrrolidone (Sigma) prepared in PBS, $2 \mathrm{mg} / \mathrm{ml}$ collagenase (Sigma), $0.025 \%$ trypsin (Gibco BRL) and $0.02 \mathrm{mg} / \mathrm{ml}$ DNase (Sigma), the contents were shaken in a $37^{\circ} \mathrm{C}$ incubator at $250 \mathrm{rpm}$ for 3 to 10 minutes. The dissociated ovary mixture was then diluted by half with Hepes-buffered MEM, pH 7.2 (Gibco BRL), modified as described [42]. A micropipette was used to draw the media solution in and out to allow further dissociation of any oocytecumulus cell complexes. Twenty-five dpp GV stage oocytes were isolated by puncturing ovarian follicles and for oocytes at this time point $50 \mu \mathrm{g} / \mathrm{ml}$ dibutyryl cyclic AMP was added to the MEM-H in order to prevent GVBD. MII oocytes were collected from 7-8 week old CD-1 females superovulated by injection of 7.5 IU of pregnant mares' serum gonadotropin (Sigma). This first injection was followed 44-48 hours later by 5 IU of human chorionic gonadotropin, (Sigma). MII oocytes were recovered 20 hours post-hCG from the oviducts and $1 \mathrm{mg} / \mathrm{ml}$ hyaluronidase was used to disperse cumulus cells (Sigma).

All oocytes were collected using a mouth-controlled drawn-out glass pipette and somatic cells were removed by transferring all oocytes through three dishes of media. Only cumulus-free and non-fragmented oocytes were chosen for experiments. Oocytes were carefully collected for all samples so that the proportion of 20 to $50 \mu \mathrm{m}$ and 60 to $80 \mu \mathrm{m}$ oocytes were similar across replicates and also reflective of the proportion of oocytes found in the ovaries at respective stages. To verify size each oocyte was measured using an eyepiece micrometer. At 5 dpp oocytes measured $10-20 \mu \mathrm{m}$ in diameter and were obtained from non-growing primordial follicles; $10 \mathrm{dpp}$ oocytes were collected from a pool of early-growing follicles ranging greatly from 20 to $80 \mu \mathrm{m}$ in diameter with roughly half measuring under $50 \mu \mathrm{m}$; while $95 \%$ of oocytes collected at $15 \mathrm{dpp}$ measured 60 to $80 \mu \mathrm{m}$ in diameter. GV stage oocytes which are fully grown $(80 \mu \mathrm{m})$ were collected at $25 \mathrm{dpp}$ and superovulated 8 week old females were used for the collection of ovulated MII oocytes. 
Because of previously described differences in the transcript levels in oocytes of different diameters, particular attention was taken in collecting Dnmt1o- and Dnmt3Lwild-type, heterozygous and homozygous oocytes to ensure that equivalent pools of oocytes were collected for the different genotypes. After washing, oocytes were measured a second time to ensure that any observed expression differences were reflective of the genotype of the oocytes and not differences in their diameters. All experiments were conducted in compliance with Canadian Council for Animal Care guidelines.

Before storage at $-80^{\circ} \mathrm{C}, 10 \mu \mathrm{l}$ of Trizol (Invitrogen) was added to oocyte samples intended for RNA extraction to prevent RNA degradation. Dnmt1o and Dnmt3L +/- and -/ - oocytes were collected using the same techniques outlined above from mice that have been previously described $[8,14]$.

\section{Real-time RT-PCR}

Oocyte total RNA was extracted using Trizol reagent according to the manufacturer's instructions and supplemented with $10 \mu \mathrm{g}$ of mussel glycogen (Boehringer Ingelheim) as previously described $[19,43]$. To serve as an internal control for RNA extraction and amplification efficiency, $0.125 \mathrm{pg}$ of rabbit $\alpha$-globin mRNA (Sigma) was added per oocyte for each sample prior to RNA extraction [21]. Oocyte RNA pellets were dissolved in RNase-free water to give a final concentration of 10 oocytes per $\mu$ l.

Total RNA was extracted from two independent collections of pooled oocytes for each QRT-PCR experiment performed. QRT-PCR was performed as previously described [26]. Briefly, experiments were carried out using the Quantitect SYBR Green RT-PCR kit (Qiagen) and performed on the Mx4000 QPCR system (Stratagene) using the standard curve method [44]. The primers used to amplify Dnmt1o, Dnmt3a, Dnmt3b, Dnmt3L, $18 S$ and rabbit $\alpha$-globin have been previously described $[19,26,43]$; the primers used to assess expression of Dnmt3a and $D n m t 3 b$ were designed to estimate the overall expression of these genes. Expression of Dnmt3a was further explored using transcript variant-specific primers designed to determine the relative expression levels of Dnmt3a and Dnmt3a2, the two major transcripts of this gene. Primer sequences and amplification conditions are described in La Salle and Trasler, 2006 [25]. The primers used to determine the expression of the Dnmt3a transcript variants by QRT-PCR analyzed the expression of each individual variant: the sense primer was unique to each transcript while the reverse primer was common to both; the expression of each variant was assessed individually, not in the same reaction tube, to give an expression profile for each variant (and not a profile for Dnmt3a and a combined profile for Dnmt3a/Dnmt3a2).
QRT-PCR was carried out using 5 to 20 oocytes per sample depending on the Dnmt gene being tested and performed in triplicate for each of the 2 independent collections of oocytes. For the developmental studies, standard curves for each experiment were established using single-use aliquots of 6 dpp testes total RNA supplemented with rabbit $\alpha$-globin, while standard curves for the studies involving the Dnmt1o and Dnmt3L +/- and -/- oocytes were established using single-use aliquots of 20 dpp ovary RNA also supplemented with rabbit $\alpha$-globin. Data were analyzed by normalizing the RNA quantity determined for the test enzyme to the rabbit $\alpha$-globin content of the same sample and plotted by calibrating to the lowest-expressing sample. Rabbit $\alpha$-globin $\mathrm{Ct}$ values were consistent across samples and replicates suggesting that our mRNA recovery rate and cDNA synthesis efficiency was uniform across experiments. Single product amplification was verified by electrophoresing QRT-PCR products through 2\% agarose gels. Results are presented as mean $\pm \mathrm{SD}$.

\section{DNA isolation and methylation analysis}

DNA was isolated from roughly 500 Dnmt3L-/- oocytes at 15 dpp and bisulfite treatment was carried out as previously described $[8,45,46]$. Nested PCR amplification for Snrpn, Igf2r, Peg3, H19, and non-nested PCR amplification for IAPs were carried out also with bisulfite specific primers and conditions that have been previously described [8,45]. An ABI 310 sequencer was used to sequence clones containing the appropriate inserts and only sequences that showed a bisulfite conversion efficiency of $>95 \%$ were used for analysis. Sequence differences between clones with similar CpG methylation profiles were verified to ensure unique clones were represented. We examined a total of $16 \mathrm{CpG}$ sites in a $419 \mathrm{bp}$ fragment of Snrpn (2151-2570 bp, AF081460), 7 CpG sites in a 205 bp fragment of Igf $2 r$ (796-1001 bp, L06446), $18 \mathrm{CpG}$ sites in a $286 \mathrm{bp}$ fragment of Peg3 (2770-3056 bp, AF105262), 16 CpG sites in a 422 bp fragment of H19 (1304-1726 bp, $\underline{\mathbf{U} 19619)}$ ) and $9 \mathrm{CpG}$ sites in a 212 bp fragment of IAP (100-312 bp, M17551). Methylation analysis of $\mathrm{H} 19$ served as an internal control for somatic cell contamination as this imprinted gene acquires methylation during spermatogenesis and should be unmethylated in oocyte samples.

For IAP and LINE-1 methylation analysis, approximately 4 ng DNA, corresponding to approximately 700 oocytes, was digested with methylation sensitive restriction enzyme HpaII and methylation insensitive isoschizomer MspI before DNA blotting with IAP long terminal repeat (LTR) or LINE-1 5' UTR probes as previously described [13]. The experiment was done in duplicate with different oocyte samples and was reproducible. 


\section{Protein extraction and immunoblotting}

Dnmt3L-wild type and -homozygous oocytes collected at 15 dpp were boiled in reducing sample buffer for $5 \mathrm{~min}$ utes and electrophoresed on an 8\% SDS-polyacrylamide gel. 280 oocytes were loaded per lane. A type B spermatogonia protein lysate prepared as previously described was also electrophoresed in parallel to serve as a positive control in the detection of DNMT3a and DNMT3b isoforms [25]. Proteins were transferred to a Hybond ECL nitrocellulose membrane (Amersham, Montreal, QC, Canada) that was blocked in 5\% non-fat dried milk. The membrane was incubated with the following primary antibody diluted in blocking buffer: clone 64B1446, a monoclonal antibody (mAb) raised against recombinant mouse DNMT3a (1:400; Imgenex, San Diego, CA). The membrane was washed according to the manufacturer's instruction (Amersham) and incubated with a horseradish peroxidase (HRP)-conjugated goat anti-mouse IgG antibody (1:1000; Vector Laboratories). After exposure to ECL Plus Western Blotting detection solution (Amersham), chemiluminescence was revealed on Hyperfilm ECL film (Amersham). The membrane was then stripped and reprobed with the following primary antibody diluted in blocking buffer: clone 52A1018, a mAb raised against recombinant mouse DNMT3b (1:400; Imgenex). India ink staining of the membrane was used to confirm loading (see Additional file 4).

\section{Statistical analysis}

SPSS software was used for statistical analysis of the QRTPCR data obtained where the number of replicates allowed for valid interpretation. Prior to performing statistical tests, the data was analyzed to determine whether it conformed to a normal distribution. Comparisons between timepoints or samples were performed using a univariate analysis of variance. For multiple comparisons, Bonferroni or Games-Howell post hoc tests were used. A p-value of $<0.05$ was taken to be significant.

\section{Abbreviations}

DNMTs DNA methyltransferases

DMRs Differentially methylated regions

ICEs Imprinting control elements

QRT-PCR Real-time RT-PCR

MII Metaphase II

\section{Authors' contributions}

DL carried out the oocyte isolations, QRT-PCRs, and bisulfite sequencing experiments unless otherwise indicated. SL was responsible for the Dnmt3L mice husbandry and genotyping, designed the QRT-PCR primers, opti- mized QRT-PCR cycling conditions, and performed the transcript-specific QRT-PCR and Western Blot analyses. DB performed the IAP Southerns. JM was responsible for the Dnmt1o mice husbandry and genotyping. THB participated in the study design. DL, SL and JMT were responsible for the study design and manuscript drafting. DB, THB and $\mathrm{JM}$ revised the manuscript and all authors read and approved the final manuscript.

\section{Additional material}

\section{Additional file 1}

Developmental expression profile of $18 S$ during postnatal oogenesis. QRT-PCR was used to determine the expression profile of 18 S in postnatal oocytes. Relative expression values obtained were normalized to the level of rabbit $\alpha$-globin expression for each sample. $18 \mathrm{~S}$ expression significantly increased between 5 and $10 \mathrm{dpp}(p<0.01)$ and again between 10 and 15 $d p p(p<0.05)$ making it an unsuitable normalizer for experiments looking at the expression level of mRNA transcripts during postnatal oocyte development. Results are presented as mean $\pm S D$.

Click here for file

[http://www.biomedcentral.com/content/supplementary/1471-

213X-7-36-S1.ppt]

\section{Additional file 2}

Developmental expression profiles of Dnmt3a, Dnmt3b, and Dnmt3L during postnatal oogenesis â $\boldsymbol{\epsilon}^{\prime \prime}$ second replicate. QRT-PCR was used to determine the expression profile of a) Dnmt3a (light grey bars) b) Dnmt3b (cross hatch bars) and c) Dnmt3L (dark grey bars) in postnatal oocytes. Relative expression values obtained were normalized to the level of rabbit $\alpha$-globin expression for each sample and were calibrated to the MII oocyte expression value. Results are presented as mean $\pm S D$. For Dnmt3a, a significant increase in expression was observed between 5 and $10 \mathrm{dpp}(p<0.05)$; while in the case of both Dnmt3b and Dnmt $3 \mathrm{~L}$, the increase between 10 and $15 \mathrm{dpp}$ was found to be significant $(p<0.05)$. Click here for file

[http://www.biomedcentral.com/content/supplementary/1471213X-7-36-S2.ppt]

\section{Additional file 3}

Dnmt3a and Dnmt3b expression in DNMT3L deficient $25 d p p$ oocytes. QRT-PCR was used to analyze the expression of the de novo DNMT enzymes in Dnmt3L heterozygous (dark grey bars) and homozygous (cross hatch bars) GV stage oocytes at $25 \mathrm{dpp}$. Dnmt3a and Dnmt3b transcripts are not significantly up-regulated in DNMT3L depleted oocytes at this time point. Samples were analyzed in triplicate and relative expression values obtained were normalized to the level of rabbit a-globin expression for each sample and were calibrated to the expression in heterozygous oocytes for Dnmt3a and Dnmt3b. Results for one experiment are presented as mean $\pm S D$.

Click here for file

[http://www.biomedcentral.com/content/supplementary/1471213X-7-36-S3.ppt] 


\section{Additional file 4}

Loading control for DNMT3A and DNMT3B Westerns. India ink staining of the membrane after probing and transfer was used to confirm equal loading for the samples shown in Figure 5. Western Blot analysis of DNMT3A and DNMT3B was carried out on growing oocytes isolated from Dnmt3L wild-type and homozygous 15 dpp females (280 oocytes/ lane). The panel on the right labelled $\hat{a} \epsilon^{\wedge} B \hat{a} \epsilon^{\mathrm{m}}$ shows blotting results for type B spermatogonia

Click here for file

[http://www.biomedcentral.com/content/supplementary/1471213X-7-36-S4.ppt]

\section{Additional file 5}

Dnmt3a, Dnmt3b and Dnmt3L expression in DNMT1o deficient 15 dpp oocytes. QRT-PCR was used to analyze the expression of the DNMT enzymes in Dnmt1o heterozygous (dark grey bars) and homozygous (cross hatch bars) $15 \mathrm{dpp}$ growing oocytes. The relative expression of Dnmt3a, Dnmt3b and Dnmt3L was not significantly changed in DNMT1o depleted oocytes at this time point. Samples were analyzed in triplicate and relative expression values obtained were normalized to the level of rabbit a-globin expression for each sample and were calibrated to the expression in homozygous oocytes for Dnmt3a, Dnmt3b and Dnmt3L. Results for one experiment are presented as mean $\pm S D$. Click here for file

[http://www.biomedcentral.com/content/supplementary/1471213X-7-36-S5.ppt]

\section{Acknowledgements}

We are grateful to Marc Toppings for technical support and to Anne Segonds-Pichon for help with statistical analysis. This work was funded by a Canadian Institutes of Heath Research grant to JMT and a NIH grant to THB. DL is the recipient of CIHR and Fonds de la recherche en santé du Québec studentships and an EMBO long-term fellowship. SL is the recipient of a CIHR studentship. JMT is a William Dawson Scholar of McGill University and a Scholar of the FRSQ.

\section{References}

I. Goll MG, Bestor TH: Eukaryotic cytosine methyltransferases. Annu Rev Biochem 2005, 74:48I-5I4.

2. Reik W, Walter J: Genomic imprinting: parental influence on the genome. Nat Rev Genet 200I, 2(I):2 I-32.

3. Robertson KD, Wolffe AP: DNA methylation in health and disease. Nat Rev Genet 2000, I(I): I I- I9.

4. Bestor TH: Activation of mammalian DNA methyltransferase by cleavage of a Zn binding regulatory domain. Embo J 1992, II (7):26I I-26I7.

5. Li E, Bestor TH, Jaenisch R: Targeted mutation of the DNA methyltransferase gene results in embryonic lethality. Cell 1992, 69(6):915-926.

6. Li E, Beard C, Jaenisch R: Role for DNA methylation in genomic imprinting. Nature 1993, 366(6453):362-365.

7. Mertineit C, Yoder JA, Taketo T, Laird DW, Trasler JM, Bestor TH: Sex-specific exons control DNA methyltransferase in mammalian germ cells. Development 1998, I 25(5):889-897.

8. Howell CY, Bestor TH, Ding F, Latham KE, Mertineit C, Trasler JM, Chaillet JR: Genomic imprinting disrupted by a maternal effect mutation in the Dnmt I gene. Cell 200I, I04(6):829-838.

9. Goll MG, Kirpekar F, Maggert KA, Yoder JA, Hsieh CL, Zhang X Golic KG, Jacobsen SE, Bestor TH: Methylation of tRNAAsp by the DNA methyltransferase homolog Dnmt2. Science 2006 , 3I I(5759):395-398.
10. Okano M, Xie S, Li E: Cloning and characterization of a family of novel mammalian DNA (cytosine-5) methyltransferases. Nat Genet 1998, 19(3):219-220.

II. Okano M, Bell DW, Haber DA, Li E: DNA methyltransferases Dnmt3a and Dnmt3b are essential for de novo methylation and mammalian development. Cell 1999, 99(3):247-257.

12. Gowher H, Liebert K, Hermann A, Xu G, Jeltsch A: Mechanism of stimulation of catalytic activity of Dnmt3A and Dnmt3B DNA-(cytosine-C5)-methyltransferases by Dnmt3L. J Biol Chem 2005, 280(14): I334|-|3348.

13. Bourc'his D, Bestor TH: Meiotic catastrophe and retrotransposon reactivation in male germ cells lacking Dnmt3L. Nature 2004, 43 I (7004):96-99.

14. Bourc'his D, Xu GL, Lin CS, Bollman B, Bestor TH: Dnmt3L and the establishment of maternal genomic imprints. Science 200I, 294(555I):2536-2539.

15. Hata K, Okano M, Lei H, Li E: Dnmt3L cooperates with the Dnmt3 family of de novo DNA methyltransferases to establish maternal imprints in mice. Development 2002, I 29(8): 1983-1993.

16. Webster KE, O'Bryan MK, Fletcher S, Crewther PE, Aapola U, Craig J, Harrison DK, Aung H, Phutikanit N, Lyle R, Meachem SJ, Antonarakis SE, de Kretser DM, Hedger MP, Peterson P, Carroll B], Scott HS: Meiotic and epigenetic defects in Dnmt3L-knockout mouse spermatogenesis. Proc Natl Acad Sci U S A 2005, I 02(I I):4068-4073.

17. Paulsen M, Ferguson-Smith AC: DNA methylation in genomic imprinting, development, and disease. J Pathol 200I, 195(I):97-II0.

18. Davis TL, Yang GJ, McCarrey JR, Bartolomei MS: The HI9 methylation imprint is erased and re-established differentially on the parental alleles during male germ cell development. Hum Mol Genet 2000, 9(1 9):2885-2894.

19. Lucifero D, Mann MR, Bartolomei MS, Trasler JM: Gene-specific timing and epigenetic memory in oocyte imprinting. Hum Mol Genet 2004, I3(8):839-849.

20. Kaneda M, Okano M, Hata K, Sado T, Tsujimoto N, Li E, Sasaki H Essential role for de novo DNA methyltransferase Dnmt3a in paternal and maternal imprinting. Nature 2004, 429(6994):900-903.

21. Temeles GL, Ram PT, Rothstein JL, Schultz RM: Expression patterns of novel genes during mouse preimplantation embryogenesis. Mol Reprod Dev 1994, 37(2): 121-129.

22. Picton $H$, Briggs $D$, Gosden R: The molecular basis of oocyte growth and development. Mol Cell Endocrinol 1998, I45(I2):27-37.

23. Bao S, Obata Y, Carroll J, Domeki I, Kono T: Epigenetic modifications necessary for normal development are established during oocyte growth in mice. Biol Reprod 2000, 62(3):616-62I.

24. Hiura H, Obata $\mathrm{Y}$, Komiyama J, Shirai M, Kono T: Oocyte growthdependent progression of maternal imprinting in mice. Genes Cells 2006, II(4):353-36I.

25. La Salle S, Trasler JM: Dynamic expression of DNMT3a and DNMT3b isoforms during male germ cell development in the mouse. Dev Biol 2006, 296(I):7I-82.

26. La Salle S, Mertineit C, Taketo T, Moens PB, Bestor TH, Trasler JM: Windows for sex-specific methylation marked by DNA methyltransferase expression profiles in mouse germ cells. Dev Biol 2004, 268(2):403-4I5.

27. Chedin F, Lieber MR, Hsieh CL: The DNA methyltransferaselike protein DNMT3L stimulates de novo methylation by Dnmt3a. Proc Natl Acad Sci U S A 2002, 99(26):16916-1692I.

28. Lees-Murdock DJ, De Felici M, Walsh CP: Methylation dynamics of repetitive DNA elements in the mouse germ cell lineage. Genomics 2003, 82(2):230-237.

29. Li JY, Lees-Murdock DJ, Xu GL, Walsh CP: Timing of establishment of paternal methylation imprints in the mouse. Genomics 2004, 84(6):952-960.

30. Walsh CP, Chaillet JR, Bestor TH: Transcription of IAP endogenous retroviruses is constrained by cytosine methylation. Nat Genet 1998, 20(2): I 16-1 I7.

31. Obata $\mathrm{Y}$, Kono $\mathrm{T}$ : Maternal primary imprinting is established at a specific time for each gene throughout oocyte growth. J Biol Chem 2002, 277(7):5285-5289.

32. Gosden RG: Oogenesis as a foundation for embryogenesis. Mol Cell Endocrinol 2002, 186(2):149-153. 
33. Howlett SK, Reik W: Methylation levels of maternal and paternal genomes during preimplantation development. Development I991, I I3(I): I 19-127.

34. De La Fuente R, Baumann C, Fan T, Schmidtmann A, Dobrinski I, Muegge $\mathrm{K}$ : Lsh is required for meiotic chromosome synapsis and retrotransposon silencing in female germ cells. Nat Cell Biol 2006.

35. Gius D, Cui H, Bradbury CM, Cook J, Smart DK, Zhao S, Young L, Brandenburg SA, Hu Y, Bisht KS, Ho AS, Mattson D, Sun L, Munson PJ, Chuang EY, Mitchell JB, Feinberg AP: Distinct effects on gene expression of chemical and genetic manipulation of the cancer epigenome revealed by a multimodality approach. Cancer Cell 2004, 6(4):36I-37I.

36. Lane N, Dean W, Erhardt S, Hajkova P, Surani A, Walter J, Reik W: Resistance of IAPs to methylation reprogramming may provide a mechanism for epigenetic inheritance in the mouse. Genesis 2003, 35(2):88-93.

37. Arnaud P, Hata K, Kaneda M, Li E, Sasaki H, Feil R, Kelsey G: Stochastic imprinting in the progeny of Dnmt3L-/- females. Hum Mol Genet 2006, I 5(4):589-598.

38. Kim GD, Ni J, Kelesoglu N, Roberts RJ, Pradhan S: Co-operation and communication between the human maintenance and de novo DNA (cytosine-5) methyltransferases. Embo J 2002 2 I(I 5):4l83-4195.

39. Margot JB, Ehrenhofer-Murray AE, Leonhardt $\mathrm{H}$ : Interactions within the mammalian DNA methyltransferase family. $B M C$ Mol Biol 2003, 4:7.

40. Suetake I, Shinozaki F, Miyagawa J, Takeshima H, Tajima S: DNMT3L stimulates the DNA methylation activity of Dnmt3a and Dnmt3b through a direct interaction. J Biol Chem 2004, 279(26):278।6-27823.

4l. Eppig J], Telfer EE: Isolation and culture of oocytes. Methods Enzymol 1993, 225:77-84.

42. Clarke HJ, Rossant J, Masui Y: Suppression of chromosome condensation during meiotic maturation induces parthenogenetic development of mouse oocytes. Development 1988, I 04(I):97-103.

43. Ratnam S, Mertineit C, Ding F, Howell CY, Clarke HJ, Bestor TH, Chaillet JR, Trasler JM: Dynamics of DnmtI methyltransferase expression and intracellular localization during oogenesis and preimplantation development. Dev Biol 2002 245(2):304-3|4

44. Bustin SA: Quantification of mRNA using real-time reverse transcription PCR (RT-PCR): trends and problems. J Mol Endocrinol 2002, 29(I):23-39.

45. Lucifero D, Mertineit C, Clarke HJ, Bestor TH, Trasler JM: Methylation dynamics of imprinted genes in mouse germ cells. Genomics 2002, 79(4):530-538.

46. Warnecke PM, Mann JR, Frommer M, Clark SJ: Bisulfite sequencing in preimplantation embryos: DNA methylation profile of the upstream region of the mouse imprinted $\mathrm{HI} 9$ gene. Genomics I998, 5 I(2): 182-190.

47. Lucifero $D$, Chaillet JR, Trasler JM: Potential significance of genomic imprinting defects for reproduction and assisted reproductive technology. Hum Reprod Update 2004, I O(I):3- I 8
Publish with Bio Med Central and every scientist can read your work free of charge

"BioMed Central will be the most significant development for disseminating the results of biomedical research in our lifetime. "

Sir Paul Nurse, Cancer Research UK

Your research papers will be:

- available free of charge to the entire biomedical community

- peer reviewed and published immediately upon acceptance

- cited in PubMed and archived on PubMed Central

- yours - you keep the copyright

Submit your manuscript here:

http://www.biomedcentral.com/info/publishing_adv.asp
BioMedcentral 